switching process always begins at specific corners of the samples (Fig. 1). After the nucleation, the magnetic domain with inverted magnetization propagates at an oblique angle with respect to the electrical current before sweeping across the entire disc. The nucleation point alternates between the four quadrants with respect to the direction of the current, controlled by the signs of the current and the external magnetic field.

These features can be concisely explained by a simple phenomenological model that takes into account two components of the spin-orbit torque, namely dampinglike and field-like torques, along with the external magnetic field and the Dzyaloshinskii-Moriya interaction intrinsic to the Co nanostructure. Although the damping-like torque is well-known to be a crucial element during spin-orbittorque-induced switching, the existence and role of the field-like component had been rather subtle and unclear. Baumgartner et al. have demonstrated that the concerted effect of the field-like spin-orbit torque, the external magnetic field and the Dzyaloshinskii-Moriya interaction determines the nucleation and the propagation of the inverted domains. Fine-tuning of these three fields by thinfilm growth technology will potentially offer a precise control of the switching and its efficiency.

The authors also report on more than 10 trillion consecutive switching events in a single sample, with each event completed at a speed of approximately $1 \mathrm{~ns}$. This appears to be sufficiently robust and fast for non-volatile magnetic memory applications, reassuring the position of spin-orbit torques as a leading contender for the future generation of magnetization switching technology.
Kei Yamamoto is at Johannes Gutenberg-Universität Mainz, Institut für Physik - FB 08, INSPIRE Group, Staudingerweg 7, 55128 Mainz, Germany. Hidekazu Kurebayashi is in the London Centre for Nanotechnology and Department of Electronic and Electrical Engineering at University College London, London WC1H 01H, UK.

e-mail:kyamamot@uni-mainz.de;

h.kurebayashi@ucl.ac.uk

References

1. Baumgartner, M. et al. Nat. Nanotech. 12, 980-986 (2017).

2. Ralph, D. C. \& Stiles, M. D. J. Magn. Magn. Mater. 320, 1190-1216 (2008)

. Nat. Nanotech. 10, 185 (2015).

4. Liu, H. et al. J. Magn. Magn. Mater. 358-359, 233-258 (2014).

5. Ikeda, S. et al. Nat. Mater. 9, 721-724 (2010)

6. Kent, A. D. \& Worledge, D. C. Nat. Nanotech. 10, 187-191 (2015).

7. Sinova, J. et al. Rev. Mod. Phys. 87, 1213 (2015).

8. Garello, K. et al. Nat. Nanotech. 8, 587-593 (2013).

9. Lee, S.-W. \& Lee. K.-J. Proc. IEEE 104, 1831-1843 (2016).

Published online: 21 August 2017

\title{
SPIN QUBITS
}

\section{Germanium-vacancy defects join the family}

The construction of complex networks of qubits that preserve good quantum coherence in spite of their mutual interactions is an essential prerequisite for the realization of a hardware implementing quantum computation algorithms. Colour centres in diamond are solid-state systems that have been proposed for the realization of such qubits. They are attractive because of the ease of controllability of the spin state where the qubit is encoded, and because of the possibility to access the spin state optically. Such optical access has already allowed the realization of coherent optical-spin interfaces - the first concrete step towards networks of photonically coupled qubits.

In this respect, special interest has been given in recent years to silicon-vacancy defects in diamond nanophotonic devices. At variance with nitrogen-vacancy defects - widely investigated colour centres in diamond - the structural inversion symmetry is preserved in silicon-vacancy defects, in turn leading to reduced sensitivity to local electric field noise and improved properties as quantum emitters of indistinguishable photons.

Now, Petr Siyushev et al. report on the control of the spin state via optical and

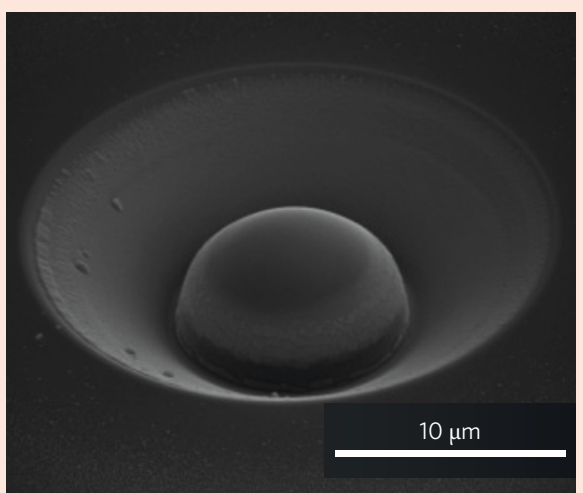

few-hours-long measurements and map the characteristic diagram of energy levels in detail, evidencing an active spin$1 / 2$ electronic degree of freedom.

Based on the observed energy levels, the researchers use two optical fields resonantly matching different electronic transitions between the Zeeman-split ground and excited states, resulting in dark spin superposition states - a condition known as coherent population trapping. Also, the irradiation of the system with properly tuned microwave radiation induces spin transitions and reshuffles the levels'

microwave fields in another diamond colour centre that preserves inversion symmetry - the negatively charged germaniumvacancy defect (Phys. Rev. B 96, 081201(R); 2017). The researchers first determine the fluorescence properties of samples polished along different crystallographic planes, improving the detection conditions by fabricating solid immersion lenses (pictured). The measurements are performed at cryogenic temperatures and also under the effect of external magnetic fields $\sim 0.3 \mathrm{~T}$. The detected optical transitions, of both spin-conserving and spin-flipping nature, are stable during populations within the ground state. With these experiments, the researchers demonstrate that the spin state of the considered germanium-vacancy defects can be controlled coherently by means of both optical and microwave fields. Both the methods reveal characteristic coherence times $\sim 20$ ns for the ground state, and - in analogy with siliconvacancy defects - the researchers identify orbital-relaxation processes mediated by phonons as the main limiting factor for this quantity.

GIACOMO PRANDO 